\title{
Too good to intervene? Thrombectomy for large vessel occlusion strokes with minimal symptoms: an intention-to-treat analysis
}

\author{
Diogo C Haussen, Mehdi Bouslama, Jonathan A Grossberg, Aaron Anderson, \\ Samir Belagage, Michael Frankel, Nicolas Bianchi, Leticia C Rebello, Raul G Nogueira
}

- Additional material is published online only. To view please visit the journal online (http://dx.doi.org/10.1136/ neurintsurg-2016-012633).

Department of Neurology and Neurosurgery, Emory University/ Grady Memorial Hospital, Atlanta, Georgia, USA

Correspondence to

Dr Raul G Nogueira, Department of Neurology, Emory University School of Medicine/Marcus Stroke \& Neuroscience CenterGrady Memorial Hospital, 49 Jesse Hill Jr Drive SE, Room 333, Atlanta, GA 30303, USA; raul.g.nogueira@emory.edu

Received 13 July 2016 Revised 5 August 2016 Accepted 10 August 2016 Published Online First 2 September 2016

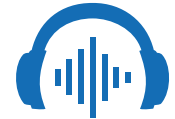

Listen to Podcast www.g0o.gl/P9v2c7

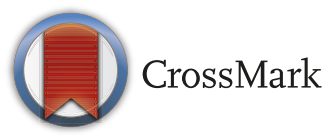

To cite: Haussen DC Bouslama M, Grossberg JA et al. J Neurolntervent Surg 2017:9:917-921.
ABSTRACT

Introduction The minimal stroke severity justifying endovascular intervention remains elusive; however, a significant proportion of patients presenting with large vessel occlusion (LVO) and mild symptoms subsequently decline and face poor outcomes.

Objective To evaluate our experience with these patients by comparing best medical therapy with thrombectomy in an intention-to-treat analysis.

Methods Analysis of prospectively collected data of all consecutive patients with National Institutes of Health Stroke Scale (NIHSS) score $\leq 5$, LVO on CT angiography, and baseline modified Rankin Scale (mRS) score 0-2 from November 2014 to May 2016. After careful discussion with patients/family, a decision to pursue medical or interventional therapy was made. Deterioration (development of aphasia, neglect, and/or significant weakness) triggered reconsideration of thrombectomy. The primary outcome measure was NIHSS shift (discharge NIHSS score minus admission NIHSS score).

Results Of the 32 patients qualifying for the study, $22(69 \%)$ were primarily treated with medical therapy and $10(31 \%)$ intervention. Baseline characteristics were comparable. Nine $(41 \%)$ medically treated patients had subsequent deterioration requiring thrombectomy. Median time from arrival to deterioration was 5.2 hours (2.025.0). Successful reperfusion (modified Treatment in Cerebral Infarction 2b-3) was achieved in all 19 thrombectomy patients. The NIHSS shift significantly favored thrombectomy $(-2.5$ vs $0 ; p<0.01)$. The median NIHSS score at discharge was low with both thrombectomy (1 (0-3)) and medical therapy (2 (0.54.5)). 90-Day mRS $0-2$ rates were $100 \%$ and $77 \%$, respectively $(p=0.15)$. Multivariable linear regression indicated that thrombectomy was independently associated with a beneficial NIHSS shift (unstandardized $\beta$ $-4.2(95 \% \mathrm{Cl}-8.2$ to -0.1$) ; p=0.04)$.

Conclusions Thrombectomy led to a shift towards a lower NIHSS in patients with LVO presenting with minimal stroke symptoms. Despite the overall perception that this condition is benign, nearly a quarter of patients primarily treated with medical therapy did not achieve independence at 90 days.

\section{INTRODUCTION}

Endovascular stroke intervention has been recently defined as the standard of care for patients with large vessel occlusion (LVO) strokes who failed to respond to, or are not candidate for, IV thrombolysis. This therapy has one of the largest effects in the neurosciences with a number-need-to-treat of only 2.5 for any reduction in disability. ${ }^{1}$ However, mechanical thrombectomy carries all the risks and expense inherent to invasive procedures. Thus, the minimal clinical stroke severity justifying endovascular intervention remains to be established. Indeed, the current American Heart Association (AHA) guidelines provide only level 1a evidence for thrombectomy for patients with baseline National Institutes of Health Stroke Scale (NIHSS) score $\geq 6$. $^{2}$ Nonetheless, a significant number of patients with mild or rapidly improving stroke symptoms on initial presentation subsequently decline and end up with poor outcomes at hospital discharge. ${ }^{3}$ In this setting, the presence of an underlying LVO is a strong predictor of neurological deterioration. ${ }^{4-6}$ The endovascular approach for low NIHSS emergent LVO has been previously reported; however, data are scarce. ${ }^{78}$ We sought to evaluate our experience with the treatment of patients with a LVO stroke and low NIHSS score by comparing best medical therapy alone with endovascular thrombectomy in an intention-to-treat analysis.

\section{PATIENTS AND METHODS \\ Patient selection}

We prospectively collected data on all consecutive patients who presented directly or were transferred to our comprehensive stroke center with an NIHSS score $\leq 5$ and an LVO on CT angiography (middle cerebral artery M1/M2, intracranial carotid artery, anterior cerebral artery (ACA), or basilar artery occlusion) from November 2014 to May 2016. Patients who were not independent (modified Rankin Scale (mRS) score >2) at baseline were excluded. The NIHSS score was assessed upon arrival by a certified neurologist and blood pressure levels recorded at the time of the initial neurological examination. This study was approved by the local institutional board.

\section{Clinical decision analysis}

All patients were evaluated upon arrival by the stroke and neurointerventional teams. A careful discussion between the treating physicians and the patients/families about the available medical evidence was routinely performed in order to decide whether intervention or medical therapy would be pursued. The decision of most patients/families was to have medical therapy, which consisted of 
initiating double antiplatelet agents (if not given IV tissue plasminogen activator) and aggressive IV hydration. In addition, the patient's blood pressure was allowed to autoregulate, and the head of the bed was kept flat. All patients were admitted to the neurological intensive care unit and monitored for deterioration with hourly neurological checks by neurointensive care nurses. Deterioration was defined as the development of deficits that could affect function (moderate/severe aphasia or neglect and/or inability to lift limbs antigravity) with worsening of the NIHSS score of at least two points; this automatically triggered reconsideration of thrombectomy. The Alberta Stroke Program Early
CT Score $^{9}$ and CT perfusion (RAPID, iSchemaView Inc, California, USA) were used to determine the ischemic core for anterior circulation strokes and perfusion defect. Hemorrhagic transformation was graded by the European Cooperative Stroke Study and reperfusion rates by modified Treatment in Cerebral Infarction (mTICI) criteria. ${ }^{10} 11$

\section{Outcome analysis}

This was an intention-to-treat analysis; patients initially receiving medical therapy alone who subsequently deteriorated and underwent endovascular therapy were analyzed in the

Table 1 Baseline characteristics of the studied groups

\begin{tabular}{|c|c|c|c|}
\hline Baseline characteristics & Thrombectomy $(n=10)$ & Medical(n=22) & p Value* \\
\hline Age (years), mean $\pm S D$ & $60 \pm 13.9$ & $68.2 \pm 14.4$ & 0.14 \\
\hline Gender (male) & $5(50)$ & $15(68)$ & 0.43 \\
\hline Hypertension & $6(60)$ & $13(59)$ & 1.00 \\
\hline Hyperlipidemia & $2(20)$ & $5(23)$ & 1.00 \\
\hline Atrial fibrillation & $3(30)$ & $8(36)$ & 1.00 \\
\hline Diabetes & $2(20)$ & $4(18)$ & 1.00 \\
\hline Smoking & $2(20)$ & $2(9)$ & 0.57 \\
\hline Anticoagulation & $0(0)$ & $0(0)$ & - \\
\hline Arterial pressure $(\mathrm{mm} \mathrm{Hg})$, mean $\pm \mathrm{SD}$ & $100.3 \pm 20.5$ & $106.7 \pm 17.7$ & 0.43 \\
\hline Baseline NIHSS score, median (IQR) & $4(2-5)$ & $2(1-4)$ & 0.09 \\
\hline Worst NIHSS score, mean \pm SD & $5.8 \pm 3.0$ & $7.1 \pm 6.8$ & 0.43 \\
\hline NIHSS score fluctuation $>4$ & $3(30)$ & $9(41)$ & 0.70 \\
\hline Wake-up stroke & $2(20)$ & $7(32)$ & 0.68 \\
\hline IV t-PA & $6(60)$ & $2(9)$ & $<0.01$ \\
\hline NCCT ASPECTS, mean \pm SD & $8.5 \pm 1.0$ & $9.1 \pm 0.8$ & 0.14 \\
\hline \multicolumn{4}{|l|}{$\mathrm{CT}$ perfusion $(\mathrm{cc})$} \\
\hline Ischemic core, mean \pm SD & $8.5 \pm 9.9$ & $0.4 \pm 1.3$ & 0.06 \\
\hline tMax $>4 \mathrm{~s}$, mean \pm SD & $132.1 \pm 111.9$ & $177.1 \pm 110.8$ & 0.39 \\
\hline $\mathrm{tMax}>6 \mathrm{~s}$, mean $\pm \mathrm{SD}$ & $65.4 \pm 52.9$ & $70.7 \pm 47.0$ & 0.82 \\
\hline $\mathrm{tMax}>10 \mathrm{~s}$, mean $\pm \mathrm{SD}$ & $24.6 \pm 23.9$ & $9.9 \pm 12.6$ & 0.16 \\
\hline \multicolumn{4}{|l|}{ Times (hours), median (IQR) } \\
\hline Last-known-normal to arrival & $6.25(3.75-9.12)$ & $10.5(4.3-15)$ & 0.19 \\
\hline Arrival to deterioration & - & $5.2(2.0-25.0)$ & \\
\hline Deterioration to puncture & - & $1.5(0.6-2.1)$ & \\
\hline Deterioration to reperfusion & - & $2.7(2.3-3.5)$ & \\
\hline Occlusion site & & & 0.62 \\
\hline ACA & $1(10)$ & $0(0)$ & \\
\hline MCA M1 & $6(60)$ & $10(45)$ & \\
\hline MCA M2 & $1(10)$ & $5(23)$ & \\
\hline Intracranial ICA & $0(0)$ & $3(14)$ & \\
\hline Extracranial ICA & $0(0)$ & $1(5)$ & \\
\hline Basilar & $2(20)$ & $3(14)$ & \\
\hline Tandem & $0(0)$ & $3(14)$ & 0.53 \\
\hline Procedure & $10(100)$ & $9(41)$ & \\
\hline Stentriever & $8(80)$ & $8(89)$ & 1.00 \\
\hline $\mathrm{mTICl} 2 \mathrm{~b}-3$ & $10(100)$ & $9(100)$ & 1.00 \\
\hline $\mathrm{mTICl} 3$ & $5(50)$ & $6(67)$ & 0.65 \\
\hline Procedure length $(\mathrm{min})$, mean $\pm S D$ & $67.1 \pm 29.6$ & $74.9 \pm 31.1$ & 0.58 \\
\hline Etiology & & & 0.19 \\
\hline Cardioembolic & $4(40)$ & $8(36)$ & \\
\hline Large vessel atherosclerosis & $1(10)$ & $7(32)$ & \\
\hline Intracranial atherosclerosis & $0(0)$ & $2(9)$ & \\
\hline Other determined & $0(0)$ & $1(5)$ & \\
\hline Cryptogenic & $5(50)$ & $4(18)$ & \\
\hline
\end{tabular}

Results are shown as number (\%) unless stated otherwise.

* Significant $p$ values are shown in bold.

ACA, anterior communicating artery; ASPECTS, Alberta Stroke Program Early CT Score; ICA, internal carotid artery; NIHSS, National Institutes of Health Stroke Scale; MCA, middle cerebral artery; mTICl, modified Treatment in Cerebral Infarction; NCCT, non-contrast CT; t-PA, tissue plasminogen activator. 
conservative therapy arm. The primary outcome measure was a change in the NIHSS score (discharge NIHSS score minus admission NIHSS score-'NIHSS shift'). This endpoint has been used previously in studies of stroke with a low NIHSS score, and has been associated with poor clinical outcome. ${ }^{12}$ For patients who died during hospitalization, the NIHSS score at day 7 was considered the discharge NIHSS score. Secondary efficacy/safety outcomes included the rates of mRS 0-2 (good outcome) at 90 days and parenchymal hematomas.

\section{Statistical analyses}

Continuous variables are reported as mean $\pm S D$ or median (IQR), as appropriate. Categorical variables are reported as proportions. Between-group comparisons for continuous/ordinal variables were made with the Student t test, Mann-Whitney $\mathrm{U}$ test, or analysis of variance, as appropriate. Categorical variables were compared by $\chi^{2}$ or Fisher exact test, as appropriate. The overall distribution of 90-day mRS was compared between groups (shift in disability levels) using the Van Elteren test (a Cochran-Mantel-Haenszel test). Scores of 5 and 6 were merged into a single group. ${ }^{13}$ Significance was set at $\mathrm{p}<0.05$ and $\mathrm{p}$ values were two-sided. Multivariable linear regression analysis for predictors of NIHSS shift was performed for variables at the 0.1 level of significance on univariate analysis (enter selection method). Statistical analysis was performed using IBM SPSS Statistics 23 (IBM-Armonk, New York, USA) except for the Van Elteren, which was computed using the FREQ procedure in SAS University Edition (SAS Institute, Cary, North Carolina, USA).

\section{RESULTS}

Thirty-two patients met the inclusion criteria over the 18-month study period and were included in the analysis; 22 (69\%) received primary medical therapy alone and $10(31 \%)$ primary interventional therapy. Baseline characteristics were comparable across the two groups (table 1), with the exception of more frequent IV thrombolysis in the primary thrombectomy group.

An orthostatic challenge was performed in 11 patients to evaluate collateral failure by elevating the patient in the stretcher and monitoring their examination. The maneuver was done for a median of $5 \mathrm{~min}$ (IQR 5-30) and led to worsening (NIHSS score $\geq 2$ points) in three (27\%) patients; one was taken for intervention and two remained in the medical group and were repositioned with head of the bed flat, without further deterioration.

A total of $9(41 \%)$ of the 22 primary medical therapy patients showed clinical deterioration and were taken for thrombectomy (figure 1). A comparison of the baseline characteristics of medically treated patients who deteriorated with those of patients who remained neurologically stable failed to indicate significant differences (see online supplementary table). The median time from arrival to deterioration was 5.2 hours (2.0-25.0); range $1-48$ hours. The median time from deterioration to puncture was 1.5 hours $(0.6-2.1)$; range $0.6-3.5$ hours. Of the four patients who deteriorated in $\leq 3$ hours, three had a good outcome, whereas of the five who deteriorated $>3$ hours after arrival, only one had a good outcome.
Figure 1 Illustrative case of a septuagenarian patient with mild aphasia treated medically who deteriorated 4 hours after presentation. (A) CT perfusion at presentation indicating no ischemic core and a sizeable perfusion (tMax $>6 \mathrm{~s}$; green) defect in the setting of National Institutes of Health Stroke Scale (NIHSS) score $=1$; (B) Frontal conventional angiography of the right common carotid upon presentation with cross-flow through the anterior communicating artery confirming an intracranial carotid artery-'I' occlusion; (C) Rescue thrombectomy with full reperfusion at $80 \mathrm{~min}$ post-deterioration after one pass of the stent retriever (inset shows the recovered thrombus); (D) final MRI diffusion-weighted imaging showing a large final infarct (modified Rankin Scale score $=5$ at 90 days).

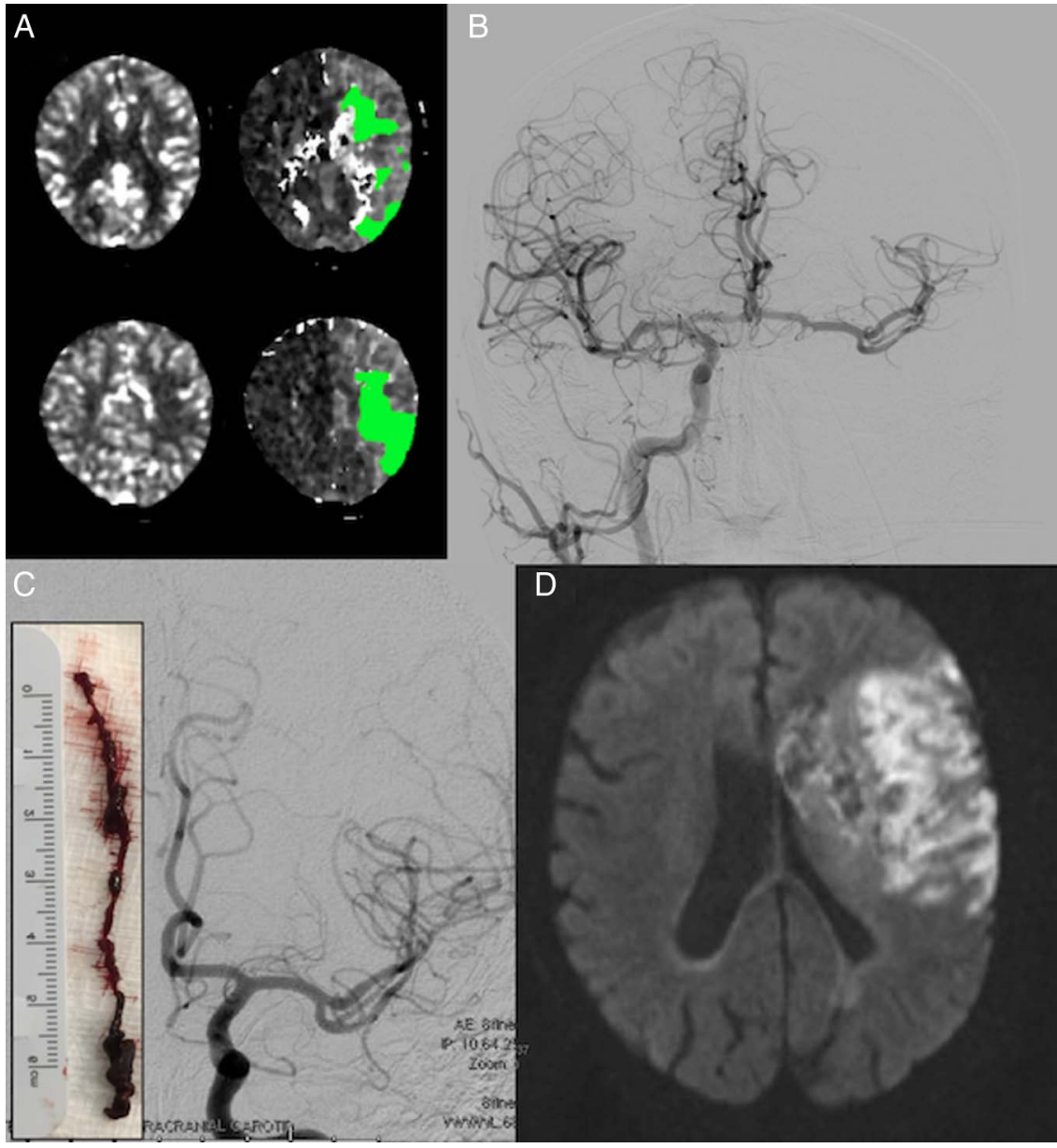


Table 2 Outcome data of the studied groups

\begin{tabular}{lccc}
\hline Outcomes & $\begin{array}{l}\text { Thrombectomy } \\
(\mathbf{n}=10)\end{array}$ & $\begin{array}{l}\text { Medical } \\
(\mathbf{n}=22)\end{array}$ & $\mathbf{p}$ Value* \\
\hline $\begin{array}{l}\text { Parenchymal hemorrhages } \\
\text { NIHSS score }\end{array}$ & $0(0)$ & $0(0)$ & 1.00 \\
$\quad$ NIHSS score at discharge, & $1(0-3)$ & $2(0.5-4.5)$ & 0.31 \\
median (IQR) & & & \\
NIHSS score worsening $>4$ /death & $1(10)$ & $5(23)$ & 0.63 \\
NIHSS shift & -2.5 & 0 & 0.01 \\
90-Day mRSt & & & 0.19 \\
0 & $4(40)$ & $5(23)$ & \\
1 & $3(30)$ & $7(32)$ & \\
2 & $3(30)$ & $5(23)$ & \\
3 & $0(0)$ & $1(5)$ & \\
4 & $0(0)$ & $0(0)$ & \\
5-6 & $0(0)$ & $4(18)$ & \\
90-Day mRS 0-2 & $10(100)$ & $17(77)$ & 0.15 \\
90-Day mortality & $0(0)$ & $3(14)$ & 0.53 \\
Discharge home & $9(90)$ & $16(73)$ & 0.38 \\
\hline
\end{tabular}

Results are shown as number (\%) unless stated otherwise.

* Significant $p$ values are shown in bold.

tThe last observation carried forward was used formissing final scores on the mRS. ${ }^{13}$ Only one patient $(10 \%)$ of the interventional group and one $(4 \%)$ of the medical therapy group had mRS carried from discharge to 90 days (both with $\mathrm{mRS}=0$ at discharge).

mRS, modified Rankin Scale; NIHSS, National Institutes of Health Stroke Scale.

Of the 19 patients undergoing thrombectomy (10 primary and 9 rescue), five (26\%) had baseline mTICI 1-2a on conventional angiography. All procedures led to successful reperfusion (mTICI 2b-3); stent retrievers were used in $84 \%$ of the cases. One procedural complication (microwire perforation) occurred in a patient treated medically who deteriorated.

Outcome data are described in table 2. The NIHSS shift significantly favored thrombectomy $(-2.5$ vs $0 ; \mathrm{p}<0.01)$. However, the median NIHSS score at discharge was low in both the thrombectomy $(1(0-3))$ and medical therapy $(2(0.5-4.5))$ groups. Good outcomes at 90 days were more frequent in the thrombectomy group $(100 \%$ vs $77 \% ; \mathrm{p}=0.15)$. Three patients in the primary medical therapy group died but none in the primary thrombectomy arm. The multivariable linear regression for predictors of NIHSS shift at discharge indicated that only thrombectomy was independently associated with a beneficial (negative) NIHSS shift (unstandardized $\beta-4.2 ; 95 \% \mathrm{CI}-8.2$ to $-0.1 ; \mathrm{p}=0.04)$. Atrial fibrillation, diabetes, and IV thrombolysis did not reach statistical significance in the multivariable analysis.

\section{DISCUSSION}

We demonstrated that endovascular therapy leads to a statistically significant shift towards a better NIHSS score upon discharge in patients with an LVO stroke and NIHSS score $\leq 5$. Moreover, a large proportion of patients with an LVO stroke and mild presentations may deteriorate despite optimized medical management.

Patients with acute ischemic stroke who are felt to be 'too good to treat' are not uncommon when presenting early. A population study indicated that $36 \%$ of patients presenting within 3 hours of stroke onset were not treated with thrombolysis owing to a mild presentation. ${ }^{12}$ Clinical deterioration is common in patients with mild stroke symptoms and underlying LVOs. ${ }^{5}$ Although IA therapy has been shown to be feasible for patients with very low NIHSS score strokes, ${ }^{78} 14$ the potential risk of harm and the associated expense may attenuate the hypothetical benefits. The best data available originate from a meta-analysis of recent randomized trials that indicates that for the subgroup of patients with an NIHSS score $\leq 10$, no benefit could be statistically demonstrated. ${ }^{1}$

In view of the overall high rate of good clinical outcomes in a population of patients with very mild presentation, we preferred not to define 'deterioration' as a small change in NIHSS score but rather as a change that could result in a meaningful degree of disability. Since the definition of clinical deterioration, poor outcome, and mild presentation varies among studies, it is difficult to make comparisons. In a study of 136 patients presenting within 24 hours of mild stroke onset, $25 \%$ deteriorated, defined by an increase of NIHSS score $\geq 4$, and $29 \%$ had poor outcomes. ${ }^{15}$ In a report of 162 patients within 6 hours of mild stroke onset, $23 \%$ had poor outcome (mRS 3-5) with LVO predicting poor prognosis. ${ }^{5}$ Another series of 41 patients who were not given IV recombinant tissue plasminogen activator owing to mild symptoms reported that $27 \%$ were not discharged home or died. ${ }^{16}$ In a study of 204 patients with mild strokes who were treated endovascularly, 62\% achieved mRS $0-3$ at 90 days. $^{7}$ Our study confirms historical data, with a rate of deterioration in the medically treated patients of $>40 \%$ and a sizeable rate of poor outcomes (despite rescue thrombectomy).

We believe that leptomeningeal collateral failure may have been the cause of the clinical decompensation in the medically treated patients who deteriorated. Repeated neurological examination in orthostasis may challenge tenuous collateralization and precipitate worsening; however, our data are too limited to allow interpretation.

This study has limitations, particularly related to its relatively small sample size and the single-center derivation. The crossover from medical therapy to thrombectomy arms might have influenced the results; however, it is unlikely that this could have affected the medical therapy arm results in light of the current data and recent interventional clinical trials. IV thrombolysis rates were higher in the treatment group; nonetheless, considering that all these patients were successfully endovascularly reperfused, the underlying use of thrombolysis should not be expected to have a significant impact, especially as thrombectomy was found to be independently associated with the primary outcome after adjustments. The fact that the occlusion site was heterogeneous makes the interpretation of the results more challenging.

\section{CONCLUSIONS}

We demonstrate a shift towards a lower NIHSS in patients with a LVO stroke presenting with mild symptoms who underwent primary thrombectomy as compared with those who received best medical therapy alone. Despite the overall perception that this condition is benign, nearly a quarter of patients primarily given medical treatment did not achieve independence at 90 days. Further studies evaluating the role of endovascular reperfusion for acute ischemic stroke with mild symptoms are warranted.

Contributors DCH: study conception, design of the work, acquisition of data, statistical analysis, interpretation of data, drafting of the manuscript. MB: data acquisition, statistical analysis, critical revision of manuscript. JAG, AA, SB, MF, NB: interpretation of data and critical revision of manuscript. LCR: data acquisition and critical revision of manuscript. RGN: study conception, critical revision of manuscript. All authors gave final approval of the version to be published, and agree to be accountable for all aspects of the work in ensuring that questions related to the accuracy or integrity of any part of the work are appropriately investigated and resolved. 
Competing interests The following authors report no conflicts of interest: $\mathrm{DCH} /$ MB/JAG/AA/SB/MF/NB/LCR: no disclosures. RGN: Stryker (PI:Trevo-2 PI/DAWN Trials), Covidien (SWIFT/SWIFT-PRIME Steering Committee, STAR Trial Core-Lab), and Penumbra (3-D Trial Executive Committee).

Ethics approval Local institutional review board (IRB)/Emory University IRB.

Provenance and peer review Not commissioned; externally peer reviewed.

Data sharing statement The unpublished data from this dataset is held by Grady Memorial Hospital/Emory University and DCH/RGN. Requests for data sharing would have to be discussed with them directly.

\section{REFERENCES}

1 Goyal M, Menon BK, van Zwam WH, et al. Endovascular thrombectomy after large-vessel ischaemic stroke: a meta-analysis of individual patient data from five randomised trials. Lancet 2016;387:1723-31.

2 Powers WJ, Derdeyn CP, Biller J, et al. 2015 American Heart Association/American Stroke Association focused update of the 2013 guidelines for the early management of patients with acute ischemic stroke regarding endovascular treatment: a guideline for healthcare professionals from the American Heart Association/American Stroke Association. Stroke 2015;46:3020-35.

3 Smith EE, Fonarow GC, Reeves MJ, et al. Outcomes in mild or rapidly improving stroke not treated with intravenous recombinant tissue-type plasminogen activator: findings from Get With The Guidelines-Stroke. Stroke 2011;42:3110-15.

4 Kim JT, Park MS, Chang J, et al. Proximal arterial occlusion in acute ischemic stroke with low NIHSS scores should not be considered as mild stroke. PLOS ONE 2013;8: e70996.

5 Nedeltchev K, Schwegler B, Haefeli T, et al. Outcome of stroke with mild or rapidly improving symptoms. Stroke 2007;38:2531-5.
6 Rajajee V, Kidwell C, Starkman S, et al. Early MRI and outcomes of untreated patients with mild or improving ischemic stroke. Neurology 2006;67:980-4.

7 Mokin M, Masud MW, Dumont TM, et al. Outcomes in patients with acute ischemic stroke from proximal intracranial vessel occlusion and NIHSS score below 8. J Neurointerv Surg 2014;6:413-17.

8 Heldner MR, Jung $S$, Zubler $C$, et al. Outcome of patients with occlusions of the internal carotid artery or the main stem of the middle cerebral artery with NIHSS score of less than 5: comparison between thrombolysed and non-thrombolysed patients. J Neurol Neurosurg Psychiatry 2015;86:755-60.

9 Hill MD, Demchuk AM, Goyal M, et al. Alberta Stroke Program Early Computed Tomography Score to select patients for endovascular treatment: Interventional Management of Stroke (IMS)-III Trial. Stroke 2014;45:444-9.

10 Zaidat 00, Yoo AJ, Khatri P, et al. Recommendations on angiographic revascularization grading standards for acute ischemic stroke: a consensus statement. Stroke 2013;44:2650-63.

11 Berger $C$, Fiorelli $M$, Steiner $T$, et al. Hemorrhagic transformation of ischemic brain tissue: asymptomatic or symptomatic? Stroke 2001;32:1330-5.

12 Willey JZ, Stillman J, Rivolta JA, et al. Too good to treat? Outcomes in patients not receiving thrombolysis due to mild deficits or rapidly improving symptoms. Int J Stroke 2012;7:202-6.

13 Saver JL, Goyal M, Bonafe A, et al. Stent-retriever thrombectomy after intravenous t-PA vs. t-PA alone in stroke. N Engl J Med 2015;372:2285-95.

$14 \mathrm{Kim}$ JT, Heo SH, Yoon W, et al. Clinical outcomes of patients with acute minor stroke receiving rescue IA therapy following early neurological deterioration. J Neurointerv Surg 2016:8:461-5.

15 Khatri P, Conaway MR, Johnston KC, et al. Ninety-day outcome rates of a prospective cohort of consecutive patients with mild ischemic stroke. Stroke 2012;43:560-2.

16 Smith EE, Abdullah AR, Petkovska I, et al. Poor outcomes in patients who do not receive intravenous tissue plasminogen activator because of mild or improving ischemic stroke. Stroke 2005;36:2497-9. 